Supporting Information:

\title{
Modulation of the Double-Helical Cores: A New Strategy for Structural Predictions of Thiolate-Protected Gold Nanoclusters
}

Wen Wu Xu, ${ }^{\dagger, \S *}$ Xiangmei Duan, ${ }^{\dagger, \S}$ Xiao Cheng Zeng***

†Department of Physics, School of Physical Science and Technology, Ningbo University, Ningbo, 315211, China

§Laboratory of Clean Energy Storage and Conversion, Ningbo University, Ningbo, 315211, China

Department of Chemistry, University of Nebraska-Lincoln, Lincoln, Nebraska 68588, United States

*Correspondence should be addressed to:*W.W.Xu (xuwenwu@nbu.edu.cn); X. C. Zeng (xzeng1@unl.edu) 
The cartesian coordinates of $\mathrm{Au}_{32}(\mathrm{SH})_{22}$

Au $1.54978200 \quad 0.49883500 \quad 1.12859800$

$\mathrm{Au}-0.17727600-0.07966800-1.38968100$

$\mathrm{Au} 1.11156600$ 2.49458400 -1.27544000

$\mathrm{Au} 0.36941300 \quad 0.85481600 \quad 3.59520200$

$\mathrm{Au} 3.18383600-1.75905900 \quad 0.70569800$

$\mathrm{Au} 1.34083400-2.41912100-1.88346000$

$\mathrm{Au}-1.48873500-2.56123900-1.59503700$

$\mathrm{Au} 2.958263000 .12046800-1.33807900$

$\mathrm{Au}-1.66431700 \quad 2.28423000-0.91917000$

$\mathrm{Au}-1.31221200 \quad 0.30676800 \quad 1.36869000$

$\mathrm{Au} 4.28105500 \quad 0.838612001 .05095600$

$\mathrm{Au} \quad 0.16727500-2.22810900 \quad 0.63904800$

Au $-0.510089001 .74619300-3.44405000$

$\mathrm{Au}-2.26196400-0.64312700-3.98533500$

$\mathrm{Au} 0.06305300 \quad 2.81801200 \quad 1.58230100$

$\mathrm{Au} 1.96776600-4.96050100 \quad 0.03078400$

Au $1.34993900-0.43420800-4.41184700$

$\mathrm{Au}-5.25791500 \quad 2.52870000 \quad 0.18829300$

$\mathrm{Au}-0.228724005 .84347300-0.54587600$

$\mathrm{Au}-3.43780800-0.13412900-0.57375200$

$\mathrm{Au}$ 3. 55192200 1. 45185900 3. 99207000

$\mathrm{Au}-0.41637700$ 4.85719800 -4.07910800

$\mathrm{Au} 3.04059900$ 3. 68070200 1. 34832100

$\mathrm{Au}-1.41109100-5.15501100 \quad 0.48288600$

$\mathrm{Au} 2.21073500-1.70609200$ 3. 69853000

Au 5. $01138000-2.73327400-2.30559400$

$\mathrm{Au}-2.58719500 \quad 0.93975800 \quad 5.06600900$

Au 6. $88834200-0.36276000-0.09711100$

S $0.15399800-6.04875600-1.06662100$

S -0.57690200 5. 190436001.70521800

S 3. 077037003.764010003 .73450200

S 3. $39388200 \quad 0.56892000-3.71084900$

$\mathrm{S}-3.353680003 .88194200-0.16597800$

S $3.01046200-3.45881600-3.34768300$

S $0.25980000-2.871412003 .05587800$

S -0.96647200 3.03099000 -5.49533300

S 3. $104472003.89978800-1.02272500$

S $4.25587300-0.769028004 .47411700$

S -4.21930400 $0.16786300-2.89767200$

S $7.17859400-2.27537700-1.45069700$

S $0.165657006 .70217100-2.72288200$

S -3. $00569700-4.42648000 \quad 2.08678400$ 
S $3.90892400-4.067245001 .07044300$

S $-0.52753600-1.58396900-5.33182300$

$\mathrm{S}-0.37355400 \quad 0.85848900 \quad 5.93383400$

$\mathrm{S}-4.799758001 .156322004 .22968800$

S 6.622533001 .552792001 .29473300

$\mathrm{Au}-2.83333100-2.128808001 .24698500$

$\mathrm{Au}-4.03245000 \quad 0.26931200 \quad 2.09133400$

$\mathrm{Au}-4.78374200-3.41145500-0.89614300$

$\mathrm{Au}-6.67201100-0.77761300 \quad 0.68873200$

$\mathrm{S}-7.35904800 \quad 1.49031000 \quad 0.56677000$

S $-3.23307500-3.97107800-2.59503200$

S -6.36319300 -3.12279600 0.85514900

$\mathrm{H}-0.64049800-1.926357003 .46350900$

H $3.93875700-0.74571700 \quad 5.80261900$

H 1.726556003 .647700003 .92004900

H 2. $429831005.09090400-1.05613300$

$\mathrm{H}-0.30304200-0.492326006 .11685900$

$\mathrm{H}-5.26915100-0.03131100 \quad 4.71202300$

$\mathrm{H}-3.705006004 .33123300-1.40579100$

$\mathrm{H}-7.736430001 .44907300-0.74553700$

$\mathrm{H}-7.48196300-3.45698900 \quad 0.14556600$

$\mathrm{H}-3.64357800-3.06475400-3.53389300$

H $-3.911214001 .49351600-2.99523000$

$\mathrm{H}-0.71676500-0.75679100-6.40179800$

H 2.94571500 1.85886500 -3.68332100

H 2. $97360200-4.69096400-2.75675900$

H 7. $65377600-1.63064600-2.55617700$

$\begin{array}{llll}\text { H } 6.84366000 & 0.90958000 & 2.47863500\end{array}$

$\begin{array}{lll}\text { H } 3.47391000 & -4.16516000 & 2.36267500\end{array}$

H $0.30941600-7.26506400-0.46397200$

H $-2.10441200-4.305268003 .10878200$

$\begin{array}{llll}\text { H } 0.62277000 & 5.64443900 & 2.17514000\end{array}$

$\mathrm{H}-0.997536007 .40504400-2.84915100$

H $0.272225002 .88725900-6.05070600$

The cartesian coordinates of $\mathrm{Au}_{40}(\mathrm{SH})_{26}$

$\mathrm{Au} \quad 0.73749600 \quad-0.95137600 \quad 0.51349100$

$\mathrm{Au}-1.98315600-1.16562400-0.83774700$

$\mathrm{Au}-0.19996000$ 1. $14609100-1.45847000$

Au 3.76148400 -0.24512200 1.72575900

$\mathrm{Au}-5.06420200-1.36579200-2.32763300$

$\mathrm{Au}-1.36104300$ 3. $84726600-3.46037000$

$\mathrm{Au}-3.66821900-3.33475600-0.44967400$ 
$\mathrm{Au}-5.26602200$ 3.63026100 -2. 15929600

$\mathrm{Au} 2.90140300-2.65087100-0.16700000$

$\mathrm{Au} 1.002530001 .58729200-4.64281300$

$\mathrm{Au}-2.48082600-3.69745700-3.17788200$

Au $1.442518003 .28236300-2.13220500$

$\mathrm{Au}-1.47594300 \quad 0.91533000 \quad 1.30739400$

$\mathrm{Au}-2.49137200-4.00926300 \quad 4.41072300$

$\mathrm{Au}-2.50129800$ 3.08042700 -0.35909900

$\mathrm{Au}$ 4. 63577800 2. 28526800 3.81881600

$\mathrm{Au} 1.110050001 .05128100$ 2.49212200

$\mathrm{Au}-0.75429400-0.85638200-3.36664600$

$\mathrm{Au} 0.37659600 \quad 3.17411500 \quad 0.45686300$

$\mathrm{Au}-0.90194800 \quad 3.32459800 \quad 3.75290100$

$\mathrm{Au}-0.58238400-1.131337003 .04116300$

$\mathrm{Au}-4.10864600 \quad 0.86917100 \quad 0.17772400$

Au $0.43976200-3.02941200-1.48489500$

$\mathrm{Au}-2.77521000 \quad 0.94241600-2.57683800$

$\mathrm{Au}-1.22874800-3.26352800 \quad 0.97572100$

$\mathrm{Au}-3.31453300-1.559503001 .69888400$

Au 2. $438943001.37098700-0.27521500$

$\mathrm{Au}-3.11813300 \quad 0.55896800 \quad 4.43984800$

$\mathrm{Au}-3.43789200 \quad 2.62954000 \quad 2.26709500$

$\mathrm{Au} 1.83326600$-3. 14930100 2. 73163900

$\mathrm{Au} 2.07221400-0.88334700-2.17817400$

$\mathrm{Au} 1.58443100-3.23654300-4.45556200$

Au 4. $77044400-0.24083300-0.84989800$

Au 6. $64600100-2.59661100 \quad 0.72347900$

$\begin{array}{llll}\mathrm{Au} & 4.95664700 & 2.05539400 & 0.74526700\end{array}$

$\mathrm{Au} 4.68711000-2.84140300-3.05485800$

S 3. $39619000-1.64465500$ 3. 72640800

S 2. 30362700 2. 433467004.16466100

S $4.61705800-4.29466500 \quad 0.46036000$

S 8. $07934800-1.08943900 \quad 0.76588900$

S 7. 01200700 2. 39903800 3. 77086900

$\begin{array}{llll}\mathrm{Au} & 7.37340900 & 0.61746400 & 2.24651600\end{array}$

S $0.36869200-4.860826001 .93175600$

S $-0.90903500-2.400144005 .12759800$

$\mathrm{S}-1.54232500 \quad 1.93127400 \quad 5.57872000$

S -2.02206300 5. $16783900-1.60051300$

S -0.09591700 4.89301100 2. 15473200

S $-0.927906002 .68708600-5.49267100$

S $-0.71800500-5.04626200-2.32297900$

S $-4.21833500-2.47824800-4.25772700$ 
S $-5.38191600-5.11242600-0.53548200$

S $-4.76199000-0.93296500 \quad 3.58236100$

S $-6.18438600-0.20378000-0.57436500$

S $-4.721442001 .92810800-3.71520900$

S -4.914167004 .142657003 .56004500$

$\mathrm{Au}-5.47027600 \quad 4.78200800 \quad 1.33363100$

$\mathrm{Au}-4.64794200-5.419427001 .70491100$

S $-3.98169800-5.798639003 .94155200$

S $-6.036868005 .46663700-0.85714600$

S $-0.15807900-1.98652700-5.47440300$

S $6.21858000-1.07523300-2.63666500$

S $3.27432100-4.67268700-3.58523800$

S 2.79945500 5.08304100-3. 11831900

$\begin{array}{llll}\text { S } 5.95912900 & 4.24440000 & 0.33072200\end{array}$

$\mathrm{Au} 4.37130500$ 4. $62387300-1.39339900$

S $3.002469000 .46940500-4.05494400$

H 4.21671400 -4.40593700 1.76333200

H $8.83981400-1.766692001 .67623000$

H 7.21752900 $1.68155600 \quad 4.91509100$

H 2. 23817800 1. $48598800 \quad 5.14483900$

H $4.51428100-2.410348003 .55687700$

H $-0.32613900-5.04171700 \quad 3.09946400$

$\mathrm{H}-1.80923900-1.53492900 \quad 5.68926700$

H $-5.07937900-5.241830004 .53187200$

$\mathrm{H}-4.58318600-6.06663100-1.09625600$

H $-1.31440300-5.37323200-1.13678300$

$\mathrm{H}-5.13860600-3.48775100-4.26150100$

H -6. $15030400-1.238878000 .31647100$

H $5.75020100-0.25909600-3.62843700$

H $3.85839500-4.98267500-4.78086100$

H $0.61587800-0.97111400-5.96550300$

H $3.509148001 .46068600-3.26053100$

H $-0.277716003 .70420800-6.13171100$

H -0.76622200 5.39092200-1. 10649400

H 3. $385285004.33031600-4.09473600$

H 5. $28034300 \quad 4.90857800 \quad 1.31156500$

H $-1.31696800 \quad 5.24683400 \quad 1.65601800$

H $-2.45053400 \quad 2.78931900$ 6. 12858300

H -5.481682000 .01190600 2. 90666000

H -5.934676003 .249038003 .71201800$

H -4.93006300 6.24357900 - 1.05127000

H -4.01220300 2.73464100 -4.56014100 
The cartesian coordinates of $\mathrm{Au}_{48}(\mathrm{SH})_{30}$ $\mathrm{Au}-1.20273400-1.384919001 .46056200$ $\mathrm{Au}-1.78470200 \quad 0.27730900-0.96966300$ $\mathrm{Au}-0.245351002 .57698600-1.48887000$ Au 1.82791600 2.89342100 -4.24034600 $\mathrm{Au} 4.79719100 \quad 0.92160300-3.98163000$ $\mathrm{Au} 5.02596800-0.70988500-0.91909100$ $\mathrm{Au} 0.43605000 \quad 0.16262300-3.15550200$ $\mathrm{Au}-2.46079200 \quad 2.05460500-3.68087400$ $\mathrm{Au}-3.67010000 \quad 0.16478300 \quad 1.35347100$ $\mathrm{Au}-1.95908100 \quad 2.59974400 \quad 0.70879100$ $\mathrm{Au}-5.16986700 \quad 4.62378200 \quad 0.97383800$ $\mathrm{Au}-7.95945000$ 3. $12514600-0.67982300$ $\mathrm{Au}-2.98869500-1.48077300$ 3. 57084000 $\mathrm{Au}-0.80340700-3.67860000-0.16363300$ $\mathrm{Au}-3.20898600-4.87759000-2.41759200$ $\mathrm{Au}-6.09328000-3.08058800-2.40568100$ $\mathrm{Au}-3.92563500-0.46250000-2.68305800$ $\mathrm{Au}-1.59300300-2.06123600-2.63232800$ $\mathrm{Au}-1.54935800 \quad 0.88206500$ 3. 14000400 S $0.335107004 .36480000-3.11302100$ S 3. $184894001.51139400-5.63581900$ S $6.589003000 .24844200-2.56315000$ $\mathrm{S}-1.07063700 \quad 0.68170900-5.04739200$ S $-3.975790003 .52534900-2.57243000$ S -3. 142454004.25583900 2. 11995600 S -7. $197699005.28495600-0.06732100$ S $-9.011954001 .14855600-1.46289300$ $\mathrm{S}-1.65809500-5.86741500-0.91647400$ S $-4.71898200-4.06628700-4.07353000$ S $-7.58060500-2.20574800-0.77387800$ S $-0.30385900-3.35641600-4.27971700$ S $-0.84270800 \quad 2.766880004 .55503900$ $\mathrm{Au} 2.485384001 .01575900-1.41794000$ $\mathrm{Au}$ 3. $00118800-0.47791500$ 1. 15115100 $\mathrm{Au} 1.17391000-0.363116003 .28872600$ $\mathrm{Au}-0.47068600-3.05811500 \quad 4.61477900$ $\mathrm{Au}-2.85868100-4.31384500 \quad 2.44385500$ $\mathrm{Au}-3.42896400-2.17268400-0.52219700$ Au $1.14035200-2.922819001 .68818100$ $\mathrm{Au} 3.59944600$-2.33811200 4.04056700 Au 5.52948500 0.56297800 3. 29361400 $\mathrm{Au} 4.61668200$ 2. $00012400 \quad 0.27086700$ 
Au 2. 63961300 1. 92870800 2. 60640700

$\mathrm{Au} 4.90650400$ 4. 87974000 2. 47930100

$\mathrm{Au} 5.775224005 .57597800-1.07036600$

$\mathrm{Au} 4.00234600$ 3.22454700 -2. 18101600

$\mathrm{Au} 2.75644000-1.38474100-2.86043700$

Au $5.94285900-3.31724100-3.14407000$

$\mathrm{Au} 7.61986800-3.51226800 \quad 0.17242000$

Au 5. $30589600-1.958535001 .58880200$

$\mathrm{Au} 3.42408400-2.90755900-0.28726700$

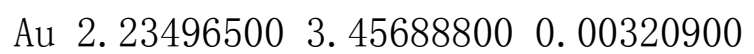

S $0.47230500-1.098749005 .55869900$

$\mathrm{S}-1.18422300-5.202273003 .87155700$

S -4.65500400 -3.66219600 1.05476000

S 2.59764800 -4.29840100 3. 14379700

S $4.57282300-0.495727005 .20220600$

S 6.674584001 .773403001 .59216500

S 3. 553064003.724237004 .03937200

S 6.411347006 .129031001 .13618500

S 5. $195907005.05946400-3.31810600$

S $4.02460500-2.80767900-4.43305300$

S $8.00739600-3.90053600-2.12492800$

$\begin{array}{llll}S & 7.33754500 & -3.04589900 & 2.48795600\end{array}$

S $2.81184000-5.11949300-1.14022300$

S 1. 103425005.351843001 .05970300

$\mathrm{Au} 0.79110400-1.38315200-0.75274500$

$\mathrm{Au} \quad 0.49172600 \quad 1.09912200 \quad 0.88682400$

$\mathrm{Au} 1.21465600-4.14897400-2.62573100$

Au 0. 201961003.92688300 2.74805000

$\mathrm{Au}-5.94326100-0.43766300-0.28686300$

$\mathrm{Au}-6.05391600 \quad 1.67632300 \quad 1.55213900$

$\mathrm{Au}-4.58201200 \quad 1.94285900-0.76752200$

S $-5.53671100 \quad 0.00453300-4.48265900$

$\mathrm{Au}-7.21595700 \quad 0.64315300 \quad-2.94321500$

S -4.65158000 - 1.798563005 .35402100

S -7. $105436001.93737300 \quad 3.73520700$

$\mathrm{Au}-5.88884300 \quad 0.03221500 \quad 4.47634500$

$\mathrm{H}-2.48989200 \quad 5.355418001 .64367300$

$\mathrm{H}-6.640141005 .56799600-1.28229900$

$\mathrm{H}-9.818604001 .78227300-2.36600300$

$\mathrm{H}-3.036574004 .20972900-1.85794600$

H $-0.309709001 .69556900-5.56386300$

H $1.268846004 .99381500-2.33783600$

H 3.90384300 2.51938900 -6.21222000 
H $6.745918001 .45279200-1.93489400$

H 6.32604400 4. $35310900-3.61361400$

H 5. 76447600 7.32885500 1.20946800

H 4.56900900 2.99127000 4. 58878700

H 2. 19595000 5. 782054001.76206100

H 0.29137800 2. 17230000 5. 02963300

H -8. $25488300 \quad 1.27271900$ 3. 41783600

$\mathrm{H}-5.35283500-2.79801700 \quad 4.74372500$

H $-7.20949300-3.07878500 \quad 0.21139600$

$\mathrm{H}-5.43567800-5.22046800-4.21784700$

$\mathrm{H}-2.51860300-6.05831100 \quad 0.12953100$

$\mathrm{H}-5.10401200-2.64452300 \quad 1.85051000$

$\mathrm{H}-2.01163900-5.48055800 \quad 4.92227300$

$\mathrm{H}-0.68671700-0.37378500 \quad 5.60367000$

H $3.53593000-4.55052500$ 2. 18130500

H $5.71155200-1.135538005 .60145200$

$\begin{array}{llll}\text { H 7. } 76734600 & 0.67859200 & 0.93789700\end{array}$

H $6.72180000-4.22060100$ 2. 81036500

H $7.81732800-5.25219100-2.10649000$

H $4.60048000-1.79357500-5.14735100$

H $3.84297000-5.18791900-2.03676300$

$\mathrm{H}-1.14035300-4.43930500-4.26847000$

H $-5.066393001 .25287500-4.78181000$

The cartesian coordinates of $\mathrm{Au}_{28}(\mathrm{SH})_{20}$ :

Au28-1

$\mathrm{Au} 4.69787700-2.95415500$ 1. 35645400

Au $1.07902100-3.064757003 .48857000$

$\mathrm{Au} 2.73565200-0.534077001 .78077100$

Au 6.63161300 -0.30800700 0.26005400

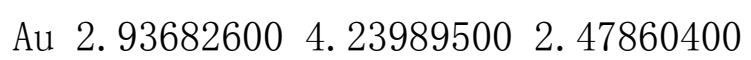

$\mathrm{Au}-2.18110900 \quad 1.92391500 \quad 2.91877000$

S 5. 134276003.632810001 .81187100

S 2. $72731900-1.449136004 .05397600$

S $6.26971500-1.44423500$ 2. 31196400

S $-0.61184100 \quad 0.27377300 \quad 3.58255500$

S 0.735366004 .851312003 .14992800

S 3. $14479700-4.56723100 \quad 0.57961000$

$\mathrm{Au} 4.15792000 \quad 1.66246700 \quad 0.75666100$

$\mathrm{Au}-0.28415600-0.97498300 \quad 1.44358300$

$\mathrm{Au} 1.42736500 \quad 1.31924000 \quad 0.03353000$

$\mathrm{Au} 3.44068600-0.43823500-0.89651200$

Au 1.40005100 -2. $88668200 \quad 0.26660900$ 
Au 0. 05291200 3. 29527700 1. 39251800

$\mathrm{Au}-1.427368001 .31922400-0.03352400$

Au $0.28418200-0.97498700-1.44357000$

$\mathrm{Au}-1.40007500-2.88664700-0.26660900$

$\mathrm{Au}-0.052930003 .29526000-1.39253800$

S $4.24198900-1.80995000-2.78588100$

S -2. $72729700-1.44912400-4.05401400$

$\mathrm{Au} 2.32099900-3.20497500-2.87829300$

$\mathrm{Au}-1.07899600-3.06473500-3.48857500$

S $0.53375800-4.76624200-3.07004500$

S - $0.735428004 .85127800-3.14994300$

S -3.69441900 3.69670200 2. 38979100

$\mathrm{Au}-2.93687900 \quad 4.23986500-2.47858000$

$\mathrm{Au}-5.44524300 \quad 2.132055002 .02851000$

S - $5.134322003 .63278300-1.81181900$

S $-0.53373400-4.766267003 .07005800$

$\mathrm{Au}-2.32098300-3.20501600 \quad 2.87828800$

S -4.24197700 - $1.80999700 \quad 2.78584600$

S $0.61185000 \quad 0.27378200-3.58254700$

S 7. $309043000.68617600-1.79080400$

$\mathrm{Au} 5.44526600$ 2. $13208100-2.02846100$

Au 2. $181119001.92392500-2.91876700$

S 3.69442900 3.69670900 -2. 38976700

$\mathrm{Au}-2.73563000-0.53405000-1.78082200$

$\mathrm{Au}-3.44065500-0.43830200 \quad 0.89647100$

$\mathrm{Au}-4.157929001 .66243900-0.75664100$

S $-3.14481900-4.56719000-0.57964900$

S -7.30900700 0.686137001 .79086200

$\mathrm{Au}-4.69791000-2.95411400-1.35647400$

$\mathrm{Au}-6.63161500-0.30799600-0.26003200$

S $-6.26974400-1.44418300-2.31197000$

$\mathrm{H}-0.76093900-5.10975800 \quad 4.37270100$

H $1.84494700-0.534179004 .55920100$

H $-3.88249400-0.930732003 .76602600$

$\mathrm{H}-1.84492300-0.53417300-4.55924500$

H $0.76096800-5.10974300-4.37268400$

H 3. $88250000-0.93069900-3.76607100$

$\mathrm{H}-5.166301004 .39779100-0.68132700$

$\mathrm{H}-0.633407003 .95279000-4.17290600$

H 0.63332600 3.95282800 4. 17289300

H 5. $16627300 \quad 4.39782300 \quad 0.68138400$

$\mathrm{H}-1.46912400-0.64315300 \quad 4.12035700$

$\mathrm{H}-4.02813800$ 4. 02332800 3. 67370500 
H $-6.81908200-0.31766200 \quad 2.57885600$

H $-7.36839100-2.25141600-2.21753300$

$\mathrm{H}-3.54418700-4.60041500 \quad 0.72832600$

H $3.54415500-4.60042400-0.72836900$

H 7. $36835800-2.25147200 \quad 2.21752200$

H $6.81913500-0.31760400-2.57883400$

H 4. $028161004.02333600-3.67367700$

H $1.46913300-0.64314100-4.12035600$

\section{Au28-2}

$\mathrm{Au}-2.58592100-0.37608400-1.76131800$

$\mathrm{Au}-1.186737001 .57405600-3.26489400$

$\mathrm{Au}-1.35431800 \quad-2.26296200 \quad 0.57722400$

$\mathrm{Au}-1.45400600-1.50416800$ 3. 25926900

$\mathrm{Au}-0.28707900 \quad 4.73032500 \quad 1.26551700$

$\mathrm{Au}-3.61411000-3.81829100 \quad 2.90960700$

$\mathrm{Au} 0.19780700-0.08001200-1.44532800$

Au $3.98402400-4.03407400-2.38994800$

$\mathrm{Au}-1.64775000 \quad 1.96525500-0.57199500$

$\mathrm{Au}-3.64930900$ 3. $49049100-2.87805700$

$\mathrm{Au}-4.81813300-2.34442500-0.16068700$

$\mathrm{Au}-5.11025600 \quad 1.38263700-0.86072300$

$\mathrm{Au}-0.25835300$ 4. $37714800-2.11471400$

$\mathrm{Au} 1.75452000-1.88234600-2.91087400$

$\mathrm{Au}-0.15663100 \quad 0.16748300 \quad 1.44504000$

$\mathrm{Au}-2.98831300-0.150683001 .31759700$

$\mathrm{Au} 1.64649300-2.16022200-0.14449300$

$\mathrm{Au} 3.04949500-0.02304600-1.23935200$

$\mathrm{Au} 0.49701500-4.73113400-1.95088600$

S - 4.27393300 -2.01002000 - 2. 44683400

S $-0.577248001 .91302200-5.60913200$

S - $1.89026000-5.069957003 .95335000$

S $-5.53509600-2.704531002 .07506700$

S $5.81085800-2.65593000-1.77136500$

S $1.950617003 .58273700-1.79143100$

S - $1.58021300-4.18135100-0.96960200$

S 1.599529006 .16846200 1. 44447300

$\mathrm{S}-2.331446005 .44576800-2.57694600$

$\mathrm{S}-2.352686003 .580621001 .16811600$

$\mathrm{S}-5.032468001 .177104001 .50269200$

S 2. $36590100-5.59711800-3.13657200$

S $-5.289216001 .79105300-3.20775400$

$\mathrm{Au} 1.43430900$ 2. $23757300 \quad 0.22186200$ 
Au 2. 63868000 0.25409800 1.79012700

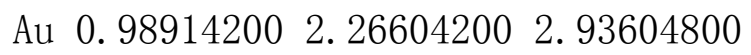
S -1.26139100 - $1.49570500 \quad 5.69362700$ $\mathrm{Au}-0.42814100 \quad 0.71652400 \quad 5.44249400$ S 0. 32305300 2. 95769800 5. 18562600 S $4.937870001 .52718900-1.51669900$ $\mathrm{Au} 4.82844400 \quad 2.23159600 \quad 0.74931000$ S 2. $04781600-3.803056001 .65679900$ $\mathrm{Au}-0.02302100$-4. 29491300 2.69320400 Au $5.00135600-1.90842300 \quad 0.33842900$ S $4.42458100-1.239029002 .54747000$ S $1.49210000-2.35294300-5.29520200$ $\mathrm{Au} 0.46173900-0.21791100-5.45161300$ $\mathrm{Au} 3.10248700$ 4.50897300 2. 22593900 $\begin{array}{llll}S & 4.85078000 & 3.07447000 & 2.98893400\end{array}$ $\mathrm{H}-4.44276600 \quad 0.79439300-3.60669600$ $\mathrm{H}-2.694324005 .66354600-1.27796700$ H $1.904098002 .55933400-2.69356200$ $\mathrm{H}-5.88736600 \quad 0.12092400 \quad 1.63533000$ $\mathrm{H}-3.43183900-3.08145800-2.55380000$ $\mathrm{H}-1.754396001 .47505700-6.14386400$ H 2. $74396800-1.96083400-5.67217900$ H $5.932225000 .60920000-1.33505900$ H 4. 03475500 2. 10938400 3.51444100 H $1.981372006 .10257600 \quad 0.13434600$ $\mathrm{H}-2.15719500 \quad 2.76563600 \quad 2.24545600$ $\mathrm{H}-0.04487900-2.11472900 \quad 5.73072300$ H 1.54290600 2.78391400 5.77291000 $\begin{array}{lll}\text { H } 5.37861700 & -0.26736900 & 2.65699900\end{array}$ H $5.39813600-1.57750500-2.50149600$ H 2. $67999800-6.59459700-2.25747700$ $\mathrm{H}-1.57549200-5.15659400-0.01659500$ $\mathrm{H}-6.29526200-3.81696000 \quad 1.85312500$ H $-2.07521800-6.236230003 .26624900$ H $2.04517900-4.88042600 \quad 0.81932200$

The cartesian coordinates of $\mathrm{Au}_{36}(\mathrm{SH})_{24}$ : Au36-1

Au 4. $01546400-0.581712001 .51631000$

$\mathrm{Au} 2.95509600-2.16151000-0.48622400$ Au $4.52885100-2.45694400-3.36339100$ Au 4. 33648100 -3. 54731500 2. 59066300 $\mathrm{Au} \quad 0.08021500 \quad 0.87690500 \quad 5.32556000$ Au 2. $45014600 \quad 0.71749000-0.50448200$ 
Au $4.98015900-0.38899700-1.11039600$

Au $1.327118000 .67786200 \quad 2.27685800$

$\begin{array}{lll}\text { Au } 1.23858300 & 4.37399200 & 2.39687700\end{array}$

Au 5. 56082500 2. $89762000-1.24837200$

Au 3. $21852100-0.54596600 \quad 4.76408100$

S $4.487061004 .80425600-0.30810500$

$\begin{array}{lll}\text { S 2. } 17101900-4.02450000 & 3.42597700\end{array}$

S $6.58313500-3.103189001 .96381500$

S - $1.266838002 .77740000 \quad 4.82506600$

$\begin{array}{llll}\text { S } 2.72199400 & 2.62864500 & 2.96251900\end{array}$

$\begin{array}{lll}\text { S } 5.22649100-0.07394400 & 3.58991300\end{array}$

S $6.703089001 .00389300-2.12345900$

S $2.83800600-1.12094900-4.32450500$

S $1.30042800-1.024463006 .08501200$

$\mathrm{Au} 1.24231600$-2. 11990900 2. 16693600

Au 2. 39572100 3. $55171300-0.52898800$

$\mathrm{Au}-0.02441500-0.845316000 .03716600$

$\begin{array}{lll}\mathrm{Au} & 0.00079300 & 2.23262900-0.12756400\end{array}$

$\mathrm{Au} 1.18487400$ 2. $13414000-2.64543400$

Au $1.08720200-0.99450100-2.59460500$

$\mathrm{Au}-1.11902200-0.65905800 \quad 2.69048000$

$\mathrm{Au}-1.17172600 \quad 2.43190000 \quad 2.38877900$

$\begin{array}{llll}\mathrm{Au} & -2.47777100 & 0.81472400 & 0.40828900\end{array}$

$\mathrm{Au}-1.32849200 \quad 0.42552900-2.33637300$

$\mathrm{Au}-1.30448700-2.35668100-1.94851200$

$\mathrm{Au}-2.35529000 \quad 3.64527700 \quad 0.14131700$

S $1.241872002 .24574200-5.10025900$

S $-5.15859200-0.55730600-3.64894200$

$\mathrm{Au}-0.049936000 .27210100-5.39916900$

$\mathrm{Au}-3.11760000-1.17107800-4.69472300$

S $-1.15790000-1.77774900-5.88573000$

S -4. $45890000 \quad 4.88823700-0.10206000$

S $-2.96217800-0.441000004 .32265400$

$\mathrm{Au}-5.53327600 \quad 3.05393300 \quad 0.97348100$

$\mathrm{Au}-4.48942300-2.07821700 \quad 3.57683300$

S -6.698067001 .248049001 .99367100$

S $2.03725800-4.40404100-0.99550100$

Au $0.00679800-4.17068100 \quad 0.23045000$

S $-2.05523400-4.25864800 \quad 1.42739400$

$\begin{array}{llll}S & -2.72367000 & 2.26594600 & -3.27028400\end{array}$

S -0. $199299006.24011300 \quad 2.03738500$

$\mathrm{Au}-0.007639005 .91407400-0.30783400$

$\mathrm{Au}-1.25124200 \quad 4.05798700-2.83436900$ 
S $0.202383005 .93982200-2.67292900$

$\mathrm{Au}-4.01679400-0.71755900-1.48128300$

$\mathrm{Au}-2.95823100-2.06757000 \quad 0.69321300$

$\mathrm{Au}-5.00150300-0.25866400 \quad 1.10321500$

S $-2.27829300-4.41613800-2.85542700$

S -6. $08390900-3.764612003 .08712700$

$\mathrm{Au}-4.44099000-3.78036100-2.12839600$

$\mathrm{Au}-6.25684900-3.33014700 \quad 0.76168800$

S $-6.67767300-3.18720300-1.56725900$

S $6.31510300-3.86911700-2.69019200$

$\mathrm{Au} 6.28428200-3.36791600-0.37358800$

H 2.24854600 -2. $11440500-5.05052300$

H $5.56451700-5.00713000-2.60272400$

H 7. $07804200-4.35854000$ 2. 18075300

H $1.79570200-4.97670900$ 2. 52051000

H 2.74760300 -5.04739000 -0.02266900

H - 1. $56211900-3.80929300 \quad 2.61763300$

H 6. $134121001.03490700-3.36432600$

H 4. $385354005.50947700-1.47260500$

H -4. 325849005.657464001 .01747200

H -6. 12351100 1. 37107000 3. 22641400

H $-2.41127000-1.17432000 \quad 5.33289500$

H $-5.17515300-4.77690400 \quad 2.95793200$

H -7. $22328000-4.43566600-1.67699900$

$\mathrm{H}-1.95345100-5.21598100-1.79556200$

H 2. 37100600 2. 54363600 4. 28109900

$\mathrm{H}-1.36352000 \quad 5.53786300 \quad 2.19605200$

H 1.36070100 5. $21156600-2.72667900$

$\mathrm{H}-3.50946500 \quad 2.41229300-2.16060400$

H $-4.99619400 \quad 0.78731000-3.83888700$

$\mathrm{H}-1.60460100-1.43973500-7.13135900$

H $0.225532003 .15298900-5.20923300$

H $-0.276799003 .71900700 \quad 4.85717000$

H $0.75145500-1.91108800$ 5. 20071200

H 5. 03394100 1.27744300 3.49843500

\section{Au36-2}

$\mathrm{Au}$ 4. $36978900 \quad 0.25796700$ - 1.11358800

$\mathrm{Au} 4.03983800-2.51349200-1.51178200$

Au 1.81330900 2. $03703400-0.99602700$

$\mathrm{Au} 0.88779300$ 3. 32434900 1. 30801300

Au 2. 85421400 0. 16451600 4. 12353900

$\mathrm{Au} 1.71085400$ 5. 35750100 -1. 14431100 
$\mathrm{Au} 1.75670300-0.94146700-1.11990300$

$\mathrm{Au}-2.81658900-0.17114900-4.14127900$

$\mathrm{Au} 3.70338100-1.39038000 \quad 0.99166800$

$\mathrm{Au} 6.74277500-3.17571900-0.09516400$

$\mathrm{Au} 4.654932003 .72818000-1.01827600$

$\mathrm{Au} 6.54330800 \quad 0.35370500 \quad 1.12492900$

$\mathrm{Au} 3.74715600-4.77539700 \quad 0.80151500$

$\mathrm{Au} 0.11357000-1.05966600-3.45408000$

$\begin{array}{cccc}\mathrm{Au} & 0.76610900 & 0.51043600 & 1.33846400\end{array}$

$\mathrm{Au} 3.26942300$ 1. 78248400 1. 38941500

$\mathrm{Au}-0.81993000 \quad 0.38325500-1.30206100$

$\mathrm{Au}-0.65310800-2.43773400-1.13171500$

$\mathrm{Au} 0.017459001 .93294000-4.21897100$

S $5.685505001 .98528700-2.26136300$

S $4.36330100-4.01621300-3.41041600$

S $-0.284794005 .34944900-2.43069600$

$\begin{array}{llll}S & 3.71847800 & 5.59199500 & 0.11951200\end{array}$

S $-3.80931300-2.14749000-3.28922400$

S $1.68476600-4.562654001 .92925200$

S 2. $235557002.21438300-3.44104100$

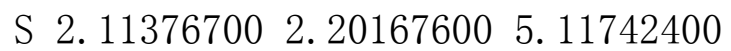

S $5.83739100-5.36724700-0.16153900$

S $3.90857600-1.839384003 .40573500$

$\begin{array}{llll}\mathrm{S} 5.46587400 & 2.00294500 & 2.45707000\end{array}$

S $-2.117317001 .81360600-5.26126600$

S $7.94269700-1.13396300-0.09823300$

Au -0.97898200 3. $17742000-1.50871800$

$\mathrm{Au} \quad 0.84176500 \quad-2.29568700 \quad 1.50223600$

$\mathrm{Au}-1.89417900 \quad 2.05408100 \quad 0.88539700$

$\mathrm{Au}-1.70690100-0.960504001 .26835600$

$\mathrm{Au}-0.14628000-0.75760500$ 3. 63488900

$\mathrm{Au}-3.345148001 .59610900-1.48531900$

$\mathrm{Au}-3.55937500-1.56417100-0.90240400$

$\mathrm{Au}-4.32172500 \quad 0.16340100$ 1. 13339600

$\mathrm{Au}-3.99936700-2.591892001 .61369500$

S $-0.94745000-1.59333500 \quad 5.79632800$

$\mathrm{Au}-2.63802900-2.794018004 .63838000$

S $-4.27371200-4.098970003 .50733500$

S $-5.538996001 .74252900-2.58803900$

$\mathrm{Au}-6.55079700 \quad 0.23907300-1.05596000$

S 0. 102459005.49914600 2. 14082400

$\mathrm{Au}-1.91027200 \quad 5.36276700 \quad 0.89142400$

S -3.93660100 5.47565700 -0.36443100 
S $-1.23432900-4.72223000-0.41734700$

$\mathrm{Au}-3.58010300-4.83685700-0.67927500$

$S-5.81213900 \quad 1.86420500 \quad 2.08808700$

S -7. $71392500-1.12381200 \quad 0.50834300$

$\mathrm{Au}-4.80010400 \quad 3.59095800 \quad 0.80211300$

$\mathrm{Au}-6.64563300-3.12003000-0.19646800$

S $-5.89697500-5.22449600-0.99483300$

S $0.84653900-1.78471900-5.68538500$

$\mathrm{Au} 2.57692600-2.92341400-4.52200900$

$\mathrm{Au}-0.04602200$ 2.21010700 4. 12828000

$\mathrm{S}-2.27608200$ 2. 35037000 3. 33699700

H $6.41119900-5.83716000 \quad 0.98603500$

H $7.53861800-0.75715600-1.34669100$

H 5. 88228200 3. 07045100 1. 71090700

H $0.94398000-5.22704900 \quad 0.99221000$

H $3.62656100-5.04537200-2.89872100$

$\mathrm{H}-0.00090300-2.85053400-5.78059600$

$\mathrm{H}-1.30055000-4.36663300 \quad 0.90016800$

H $-5.91556400-4.87262100-2.31501500$

H $-8.84902800-1.31863200-0.22746200$

$\mathrm{H}-5.945718002 .87149500-1.93160300$

$\mathrm{H}-2.69512500-2.93416000-3.34255400$

$\mathrm{H}-2.75230900 \quad 2.67519200-4.41288300$

H 2.64879500 $0.92584200-3.62968100$

H $6.790908001 .86043200-1.46851900$

H 4. $289547006.55837000-0.65995600$

H $0.257200004 .86189900-3.58756000$

H -0.39491400 5. $03838200 \quad 3.32848200$

$\mathrm{H}-3.447266004 .79056300-1.44217900$

$\mathrm{H}-5.09631100$ 2. 12200700 3. 22432300

H $-5.36548700-3.46926700 \quad 4.03173500$

$\mathrm{H}-1.70531100-0.503069006 .11401700$

H 5. $18802500-1.376907003 .53317400$

H 1.72542900 1.66527500 6. 31214600

H -2.59068300 1.03970100 3.56703300

The cartesian coordinates of $\mathrm{Au}_{44}(\mathrm{SH})_{28}$ :

Au44-1

$\mathrm{Au}$ 5. $44892100 \quad 0.45140300-0.22890700$

$\mathrm{Au} 3.07432300 \quad 0.31027500 \quad 1.38887500$

$\begin{array}{llll}\mathrm{Au} & 0.87170100 & 0.34895100 & 3.17842100\end{array}$

Au 3. $09593000-4.282031001 .68313600$

$\mathrm{Au} \quad 0.52535600 \quad-3.84238100 \quad 4.02189700$ 
$\mathrm{Au} 3.82263800$ 4. 97519600 2. 05820000

$\mathrm{Au} 3.29923100-1.65265100-0.86447600$

$\mathrm{Au} 1.54621000$ 2.83846000 -3.21940400

$\mathrm{Au} 5.13129800$ 2. 14232900 1.99240500

Au 3. $56289000-3.98742900-3.24742000$

Au 3. $48991400 \quad 0.50384300-2.65120700$

$\mathrm{Au} 0.95765100-1.682438001 .17593400$

Au 2. $72721100-1.74374700$ 3. 31354200

Au 3.67804700 2.58184300 -0.31191700

$\mathrm{Au} 1.20678100 \quad 0.45719400-0.94950100$

$\mathrm{Au} 6.01114600-1.75027000-2.81680800$

Au $1.55421900-1.52010700-3.02934800$

$\mathrm{Au} 5.28431500 \quad 0.04696900 \quad 4.60021200$

Au 6. 43302800 2.35700400 -2.52456800

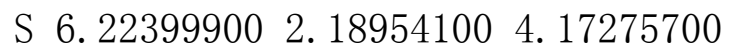

S 1.64244100 4.24980500 2.59070600

S 5.95739000 5.98937000 1.80340400

S $0.299782001 .61358900-4.85747200$

S $7.26582000-1.03801100-0.92972900$

S 2. $28828800-5.50280900-1.94245400$

S $4.889291001 .78071600-4.23104700$

S 4. $38231200-2.09326500$ 5. 10149300

$\mathrm{S}-0.76695400-2.27838000 \quad 5.24979400$

S $4.90386400-2.66248500-4.71536300$

S $1.60504000-5.654751002 .92474900$

S $4.77498700-3.176139000 .43681700$

S 2. $743117004.32190400-1.78709000$

Au $0.91109700 \quad 2.457994001 .04467200$

Au $1.05352600-3.69103700-0.82177900$

$\mathrm{Au}-1.18329100 \quad 0.50424700 \quad 0.92757500$

Au -0.95451300 -1.71529800 - 1.10461700

$\mathrm{Au}-1.08909500-3.62575900 \quad 0.98292500$

$\mathrm{Au}-1.56784400-1.374960003 .09065300$

$\mathrm{Au}-0.85950700 \quad 2.43056700-1.12767100$

$\mathrm{Au}-0.85927600 \quad 0.24224700-3.18236300$

$\mathrm{Au}-3.06177500 \quad 0.28291200-1.39995100$

$\mathrm{Au}-3.31255800-1.57671900 \quad 0.92837200$

$\mathrm{Au}-3.47141600 \quad 0.65455400 \quad 2.61534500$

$\mathrm{Au}-2.71449100-1.84876700-3.24875100$

$\mathrm{S}-2.35135900-5.36090300 \quad 2.19248500$

S $-7.38505400-0.73152800 \quad 1.04651300$

$\mathrm{Au}-3.62197800-3.76490000 \quad 3.40169000$

$\mathrm{Au}-6.08655400-1.49488100$ 2.88303000 
S -4.92422300 -2.32121200 4.78370000

S - $4.36324700-2.34720500-5.01194900$

S - $1.523190004 .25466500-2.66486900$

$\mathrm{Au}-5.30379400-0.20801500-4.59352700$

$\mathrm{Au}-3.77085700$ 4. $85911200-2.28180800$

$\mathrm{S}-6.200020001 .97038800-4.28633900$

S - $0.288252001 .79320800 \quad 4.79233200$

$\mathrm{Au}-1.50452600 \quad 2.96795700 \quad 3.09412900$

S -2.67773900 4.40638100 1.59609300

S $-4.79583500-3.09897800-0.37903500$

S $0.73583200-2.50744900-5.14898000$

$\mathrm{Au}-0.54417900-4.01769900-3.84022100$

$\mathrm{Au}-3.11982500-4.32882400-1.51080200$

S - $1.62340000-5.77635700-2.65553400$

$\mathrm{Au}-5.43748000 \quad 0.50740900 \quad 0.21390200$

$\mathrm{Au}-3.64163600 \quad 2.63118400 \quad 0.17976500$

$\mathrm{Au}-5.12954700$ 2. $07348600-2.08475700$

S -4.82823200 $1.98789300 \quad 4.17643700$

S -5.96474600 5.76472600 -2.17122400

$\mathrm{Au}-6.41174000 \quad 2.49390000 \quad 2.48752600$

$\mathrm{Au}-6.92281700 \quad 4.24125100-0.63027900$

S -8. $15361800 \quad 2.96777000 \quad 0.94416700$

S 8. $157616002.86114500-0.96753800$

$\begin{array}{cccc}\mathrm{Au} & 6.92520800 & 4.32992600 & 0.42151900\end{array}$

H 3. $20314900-5.68587600-0.94245900$

H $3.97891800-1.66798900-4.87167100$

H $8.01515200-0.07411800-1.53986400$

$\mathrm{H}-0.23994900-1.57817200-5.37724100$

H $-2.49216600-6.14049300-3.64519400$

$\mathrm{H}-5.03399400-2.20517200-1.38636200$

H $-5.21476000-3.06916300-4.22644000$

$\mathrm{H}-7.421676001 .57197900-3.82615900$

$\mathrm{H}-0.684748002 .54832100-5.00220600$

H 3. $849711004.46249100-2.57502000$

$\mathrm{H}-0.946571005 .26678600-1.95566000$

H -6. 42217100 5. $15832300-3.30590500$

$\mathrm{H}-8.68817800 \quad 4.03986400 \quad 1.60133700$

H $-5.52465000 \quad 0.93839300 \quad 4.70742300$

H -7.21202500 - - $1.85854200 \quad 0.29147700$

$\mathrm{H}-5.86155100-3.24056800 \quad 5.16122500$

H -3. $25960400-5.591713001 .19642100$

H 5. $13065600-2.267112001 .39272600$

H 2. $48191100-5.962294003 .92618100$ 
H $0.21182000-1.345098005 .44712800$

H $5.26894400-2.825932004 .36649500$

H 5. 25951100 2. 91641700 4. 80949200

H $0.70561000 \quad 2.72354800 \quad 4.90033300$

H -3. $78131400 \quad 4.59942800$ 2. 37689000

H $0.970139005 .26927000 \quad 1.98402100$

$\begin{array}{llll}\text { H } 5.59738000 & 6.83701200 & 0.79465500\end{array}$

H 8. $789820003.82930400-1.69460200$

H $5.62455400 \quad 0.72327200-4.69002600$

\section{Au44-2}

$\mathrm{Au}$ 5. 37286800 0. 01300600 1. 30717500

$\begin{array}{llll}\mathrm{Au} & 2.52499900 & 0.06485100 & 2.46274300\end{array}$

Au 3. $272712001.34942200-0.12522900$

$\mathrm{Au} 2.29054900 \quad 3.86695000 \quad 0.78419600$

$\mathrm{Au} 1.44657700-1.03110300-0.10245500$

Au $0.43351300-2.12223800-2.50661400$

Au 0. $454389001.97031600-4.93147600$

Au 2. $50154900-4.06481200-1.01065700$

$\mathrm{Au} 0.539175001 .71604500 \quad 0.77995900$

$\mathrm{Au}-2.48720000-1.647359003 .82404000$

Au 1.42813100 2.86645500 - 1.67767500

$\mathrm{Au} 1.57179400$ 3. 42921200 3. 69341000

$\mathrm{Au} 6.80132000-2.753313001 .17479100$

$\mathrm{Au} 5.71255900 \quad 0.03644300-3.95556700$

$\mathrm{Au} 5.927793001 .32396300-1.10822200$

Au 3. $35457100-2.415648001 .45808000$

$\mathrm{Au} 4.56809000-1.07896100-1.11554600$

$\mathrm{Au}-0.49032800 \quad 5.17263600 \quad 1.46159500$

$\mathrm{Au}-0.38903300 \quad 0.66663300 \quad 3.23542700$

$\mathrm{Au}-0.54543100 \quad 0.43969700-1.81549200$

$\mathrm{Au} 2.15251900 \quad 0.08716600-2.64941000$

$\mathrm{Au} 0.74289800 \quad-2.09071600$ 2.49269400

$\mathrm{Au}-1.42089100 \quad-0.57356400 \quad 0.89065800$

$\mathrm{Au}-2.15920300 \quad 2.08590300 \quad 1.59150800$

$\mathrm{Au} 4.92141800-1.328122004 .40341000$

$\mathrm{Au} 1.71564200-1.12751300$ 5. 43174600

S 5. $03140800-4.192185001 .80216600$

S 3. 934018005.661562001 .14753300

S $0.82274800-5.20110900 \quad 0.24795100$

S $4.28134900-3.20999700-2.34487400$

S $-4.24897200-0.049139004 .00493800$

S - $1.173224005 .48017600-0.78956200$ 
S - $0.01538500 \quad 0.48723400 \quad 5.66577700$

S 3.40416300 -2.81732800 5. 46602800

S $6.45403600 \quad 0.242856003 .50371700$

S $0.166327000 .00550900-6.24300400$

S $7.81453000-1.06638900-3.92425300$

S -0. 140279005.067153003 .80738300

S $1.090408003 .98502300-3.85442500$

S 3. $664118001.15853100-4.28792300$

S $-0.83738600-3.374807003 .89189500$

S 3. 380052001.924732003 .86100900

$\mathrm{Au}-0.68494400-3.25351700 \quad 0.12501800$

$\mathrm{Au}-1.482483003 .05539400-1.17994700$

$\mathrm{Au}-2.49036700-1.94371900-1.54894500$

$\mathrm{Au}-3.29602000 \quad 0.87022100-0.95735500$

$\mathrm{Au}-2.329459001 .73260200-3.49598400$

$\mathrm{Au}-3.30234300-2.69788700 \quad 1.02593000$

$\mathrm{Au}-4.55512800 \quad 0.20160400 \quad 1.57053200$

$\mathrm{Au}-5.34240400-1.15185600-0.72099700$

$\mathrm{Au}-5.96359000 \quad 1.56202900-0.37373200$

S -3. $869848002.70745500-5.14498400$

$\mathrm{Au}-5.53998300 \quad 2.84077600-3.45964700$

$\mathrm{S}-7.259555003 .03353200-1.82932700$

S -4.89558300 -4.08878100 2.27694700

$\mathrm{Au}-6.72801700-2.70862500 \quad 1.68783900$

S $0.11498600-4.11998700-3.91616500$

$\mathrm{Au}-1.54347700-4.99293700-2.45797900$

$\mathrm{S}-3.12001800-6.09471600-1.05501000$

S -3. 619683004.040508001 .95699500

$\mathrm{Au}-5.84048300 \quad 3.26868400 \quad 2.17120100$

S -6.53496500 -2.92261600 - 1.93902300

S $-8.73524600-1.594760001 .07023000$

$\mathrm{Au}-4.78037000-4.45143400-1.46250500$

$\mathrm{Au}-8.23985100 \quad 0.62398400 \quad 1.72695800$

S -8. 146149002.845096002 .55325700

S 7. $014697003.42301000-1.69639400$

Au 5. $457712004.51306000-0.26774500$

S 8.67738600 - $1.35856500 \quad 0.71222000$

Au 8. $08667000-1.11632900-1.57273500$

$\mathrm{Au}-1.63255800-0.83223200-4.94673700$

S $-3.47060500-1.81079000-3.83824200$

$\mathrm{H}-6.11252800-2.59721200-3.19750300$

$\mathrm{H}-3.55949600-6.99905600-1.98031200$

H $-0.72950600-3.52522800-4.81396000$ 
H -4.28627000 $1.53722100-5.71096500$

$\mathrm{H}-6.84167600 \quad 4.21494200-1.28792300$

H $8.023439003 .32931700-0.78102400$

$\begin{array}{llll}\text { H } 3.44747000 & 6.50479500 & 0.19076100\end{array}$

H $-4.15107400-0.64541000-3.61441600$

$\mathrm{H}-0.57922000 \quad 0.56038000-7.24277500$

$\mathrm{H}-0.175643004 .42711700-3.59658400$

$\mathrm{H}-3.70924100 \quad 4.37332100 \quad 0.63669700$

H -8. $02256600 \quad 2.51569900 \quad 3.87306400$

$\mathrm{H}-8.42652700-1.45827700-0.25295500$

$\mathrm{H}-4.61418700-3.54122800 \quad 3.49819000$

$\mathrm{H}-5.26997100-0.94454800 \quad 4.15368800$

$\mathrm{H}-0.27395400-3.00872600 \quad 5.08510900$

H $0.78629300 \quad 1.59060300 \quad 5.77512700$

H $3.00751300-3.45369700$ 4. 32315800

H 7. $48974800-0.60745600 \quad 3.24569400$

$\begin{array}{llll}\text { H } 4.17261400 & 2.50741500 & 2.91286500\end{array}$

H $0.703973006 .13545600 \quad 3.91106800$

H $0.077942005 .53015400-1.33363100$

H 3. $49471700-2.69653400-3.33456000$

H $1.32805700-4.906569001 .48313000$

H $4.83763400-4.80759000 \quad 0.59672500$

H 9. $60350600-2.34514400 \quad 0.52259700$

H 7. $30429900-2.33065300-4.01713600$

H 3. $26423300 \quad 0.42356000-5.36630700$

The cartesian coordinates of $\mathrm{Au}_{44}(\mathrm{SH})_{28}$ :

Au52-1

$\mathrm{Au}-6.44982400 \quad 0.03989700 \quad 0.38295900$

$\mathrm{Au}-4.02264700-1.144878001 .23619500$

$\mathrm{Au}-4.11122100 \quad 1.80126600 \quad 0.97597700$

$\mathrm{Au}-6.28332300 \quad-2.72051600 \quad 0.69010300$

$\mathrm{Au}-4.78137300 \quad 1.51999300-1.71312900$

$\mathrm{Au}-1.65601700 \quad 0.31412600 \quad 1.99629400$

$\begin{array}{llll}\mathrm{Au} & 0.63023900 & 1.68364600 & 2.79218900\end{array}$

$\mathrm{Au}-6.91065500 \quad 3.14862800 \quad 0.02309400$

$\mathrm{Au}-0.00020600 \quad 4.93671700 \quad 0.00287800$

$\mathrm{Au}-0.54771900-0.02685300 \quad 5.18781900$

$\mathrm{Au}-2.45617200 \quad 0.20175200-0.85948300$

Au $0.00005900-4.39546000-0.00352300$

$\mathrm{Au}-2.537209003 .04917400-0.94500000$

$\mathrm{Au}-5.07756000-1.49947000-1.45022500$

$\mathrm{Au}-4.24617500 \quad 4.94488300 \quad 0.75997300$

$\mathrm{Au}-3.15546400-0.98037500 \quad 3.91392100$ 
$\mathrm{Au}-3.32453700 \quad 0.16414700-3.97846500$

$\mathrm{Au}-2.37928000-2.63346000-0.74920100$

Au 0.000324001 .721872000 .00109500

$\mathrm{Au}-1.608422003 .106637001 .91074100$

$\mathrm{Au}-1.68764200-2.48522600$ 2. 11034700

$\mathrm{Au} \quad 0.00013600-1.20924100-0.00136900$

$\mathrm{Au}-7.85627400 \quad 0.57678300-2.27701800$

$\mathrm{Au}-0.95111100-1.26135100-2.69339700$

$\mathrm{Au}-5.80189600-2.910005003 .99402200$

$\mathrm{Au}-5.40831000-4.70106100-1.45127400$

S -4.62816400 - $1.76259100 \quad 5.72676700$

S $0.53971500-2.141311004 .99808900$

S - 1. $30399600 \quad 2.14407000 \quad 5.88088000$

S $0.65018700-4.71419900-2.30520700$

S -6. $451853002.07439000-3.46679900$

$\mathrm{S}-2.336361002 .27912600-4.49512500$

S $-3.32872800-4.90344600-0.35994800$

S $-7.44355200-4.78319400-2.67294400$

S $-7.01328600-4.255604002 .44262800$

S 1.525719005 .127421001 .83885300

S $-5.819888004 .98809200-1.04635900$

S $-9.46855400-0.78871700-1.15259800$

S $-4.54090900-1.89136900-3.84493200$

$\mathrm{S}-1.525924005 .12933900-1.83305400$

$\mathrm{S}-2.68932500 \quad 5.25426700 \quad 2.53603200$

S -8.24685000 1.547727001 .19266000

S $-0.64980200-4.717228002 .29781600$

Au $0.95140100-1.26474800$ 2. 69043300

$\mathrm{Au}-0.63005800 \quad 1.68695200-2.78996900$

$\mathrm{Au} 2.45693200 \quad 0.20092300 \quad 0.85921600$

Au $1.65654800 \quad 0.31665000-1.99611400$

Au 1.60852100 3. $10916300-1.90696600$

$\mathrm{Au} 2.53732500 \quad 3.04837700 \quad 0.94854700$

$\mathrm{Au} 2.37944700-2.63440800 \quad 0.74450600$

$\mathrm{Au} 1.68790700-2.48243400-2.11495700$

Au $4.02334600-1.14395500-1.23868700$

$\mathrm{Au} 4.111370001 .80240500-0.97390600$

$\mathrm{Au} 4.78157600 \quad 1.519232001 .71510500$

$\mathrm{Au} 3.15579000-0.97437100-3.91596500$

S 2.68901500 5.25768300 -2.52984400

S 8. $247030001.54928400-1.18954700$

Au 4. $24595100 \quad 4.94625100-0.75418000$

$\mathrm{Au}$ 6.91065600 3.14917500 -0.01888100 
S 5.81967900 4.98757500 1.05216700

S $4.62957100-1.75635200-5.72795100$

S 3. $32886500-4.90398800 \quad 0.35254000$

$\mathrm{Au} 5.80232800-2.90540300-3.99561000$

$\mathrm{Au} 5.40727900-4.703528001 .44640200$

S $7.01334800-4.25301800-2.44564900$

$\begin{array}{llll}\mathrm{S} & 2.33653800 & 2.27479500 & 4.49762200\end{array}$

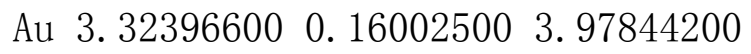

S $4.53952700-1.895824003 .84276600$

S 5. $212094001.98659800-3.21956000$

S $-0.53924800-2.13469100-5.00223100$

$\mathrm{Au} 0.54838100-0.02000800-5.18852800$

Au 3. $146772001.97013700-4.38082600$

S $1.304584002 .15210500-5.87790500$

$\mathrm{Au} 6.44966300 \quad 0.04045100-0.38259000$

Au 5. $07634900-1.500112001 .44873300$

Au $6.28352300-2.71982800-0.69135200$

S 6. 452002002.07010400 3. 46983000

$\begin{array}{llll}S & 7.44115600 & -4.78744500 & 2.67029300\end{array}$

$\begin{array}{llll}\mathrm{Au} & 7.85534100 & 0.57267700 & 2.27849500\end{array}$

Au 8. $20744500-2.715089001 .78096500$

S 9.46704400 -0.79234000 1. 15260700

$\mathrm{Au}-8.20894700-2.71110600-1.78212000$

S $-5.211423001 .98197900 \quad 3.22220200$

$\mathrm{Au}-3.14612800 \quad 1.96392400 \quad 4.38346300$

H -5.47756800 0.65250600 3. 32932700

$\mathrm{H}-1.96380100 \quad 1.74463900 \quad 6.99779700$

$\mathrm{H}-0.54698500-2.89039700 \quad 4.67911800$

$\mathrm{H}-5.41031500-0.65565900 \quad 5.77313500$

H $-8.22207700-3.678321002 .65368300$

$\mathrm{H}-5.72119200-1.39957500-4.30215500$

$\mathrm{H}-1.633743001 .86552100-5.58145000$

$\mathrm{H}-7.72316800 \quad 1.82744800 \quad 2.41465400$

$\mathrm{H}-6.62049600 \quad 5.96089800-0.53989500$

$\mathrm{H}-3.46247800 \quad 4.76191900 \quad 3.53790800$

$\mathrm{H}-5.966274001 .20712800-4.39272100$

$\mathrm{H}-10.35759800-0.88707700-2.17491200$

$\mathrm{H}-6.92960400-4.27709300-3.82214700$

$\mathrm{H}-2.67677200-5.51643000-1.37867200$

H $0.54732100-2.88432500-4.68406700$

H $1.964513001 .75461300-6.99544500$

H $5.47804000 \quad 0.65725500-3.32867400$

H $5.41223900-0.64975500-5.77354800$ 
H $8.22222900-3.67566500-2.65600600$

H $1.76875300-5.42692900-2.01781000$

$\mathrm{H}-1.76832300-5.429704002 .00961800$

H 2.67575600 -5.51844000 1.36964900

H $6.92621900-4.281762003 .81923800$

H 10. $35645500-0.89159800$ 2. 17452200

H 5. 96551200 1.20223200 4. 39471300

H 7. $724179001.83059900-2.41151900$

$\begin{array}{llll}\text { H } 6.62032800 & 5.96087800 & 0.54672900\end{array}$

H 3. $462209004.76670500-3.53236500$

H 2. 392193005.978520001 .22962000

H -2. $392556005.97972400-1.22305800$

H 1.634110001 .860185005 .58367500

H $5.71993400-1.40497300$ 4. 30068400

\section{Au52-2}

$\mathrm{Au}-4.43534100 \quad 0.58075500 \quad 0.77485400$

$\mathrm{Au}-4.03570200 \quad 0.36231000-2.18285100$

$\mathrm{Au}-2.16582500-1.03686900-0.32057800$

$\mathrm{Au}-2.12965700 \quad 1.97235500-0.35402900$

$\mathrm{Au}-6.43667100-0.84651000-0.71382000$

$\mathrm{Au}-4.02576100-2.42230300-1.97700700$

$\mathrm{Au}-1.63291800 \quad 1.96031500-3.14630800$

$\mathrm{Au}-0.19329000 \quad 0.44610500 \quad 1.47964800$

$\mathrm{Au} 1.63175500 \quad 1.80414600 \quad 3.22605600$

Au 2. $05912800 \quad 5.55395800 \quad 0.27241100$

$\mathrm{Au}-0.70380200 \quad 0.39509700 \quad 4.79459900$

Au $0.19450600 \quad 0.51735700-1.47175300$

$\mathrm{Au} 2.25197000 \quad-4.36685000 \quad 0.12268800$

$\mathrm{Au} 0.035222003 .40685100-1.43401800$

$\mathrm{Au}-6.41129900-1.15609000-4.08454700$

$\mathrm{Au}-5.63595500-2.41464200 \quad 4.26403300$

Au -1.84610500 -1.08618000 -3.16916000

$\mathrm{Au}-4.84964400-2.296509001 .04870800$

$\mathrm{Au}-8.16467800-3.47583300 \quad 1.98029200$

$\mathrm{Au}-2.06016800 \quad 5.57092900 \quad 0.02313900$

$\mathrm{Au}-4.26432800 \quad 3.42269200 \quad 0.64892300$

$\mathrm{Au}-2.48557600 \quad-0.97445500 \quad 2.51018700$

$\mathrm{Au}-2.62604100 \quad 2.03691800 \quad 2.43749100$

$\mathrm{Au} 0.70508900 \quad 0.62967800-4.77559300$

Au $0.39455000-2.35970900-1.51002400$

$\mathrm{Au} 2.13428600 \quad 1.94984900 \quad 0.43673200$

$\mathrm{Au}-6.69885300-0.51675500$ 2. 05943300 
$\mathrm{Au}-0.02556900 \quad 3.33669800 \quad 1.57866900$

$\mathrm{Au}-0.39361600-2.432901001 .37396000$

$\mathrm{Au} 2.16596400$-1. 05197000 0. 25863100

$\mathrm{Au}-3.69230400 \quad 0.34439900-5.47927400$

Au 2. $48937600-0.84916900-2.56572600$

$\mathrm{Au}-7.08980200-3.88818900-1.31485800$

$\mathrm{Au}-2.24014200-4.35375800-0.37689100$

S $-3.78557900-0.99997400 \quad 4.62753100$

S -8. $13702100 \quad 0.91333100 \quad 3.41452200$

S $0.39514800-1.705578005 .04711200$

S - $1.98677700 \quad 2.41445600 \quad 4.78235300$

S $3.80932800-4.42698500-1.68521000$

S $-2.493921002 .39043600-5.42162500$

S $1.996554002 .64120900-4.66769100$

S $-0.70566500-4.46610500-2.20214600$

S $-3.81304800-4.512818001 .40895400$

S $-5.16505900-4.53058900-2.53948000$

$\begin{array}{llll}S & 2.79498500 & 5.86890500 & 2.51511100\end{array}$

S -2.77992900 6.00517300 -2. 20363400

S $-4.83511300-1.72792000-5.77001400$

S $-0.40619400-1.45055800-5.13176600$

S $1.141894005 .56767500-1.91200700$

S -1. $13778400 \quad 5.46181300$ 2. 20303800

S $0.72099400-4.573626001 .94409600$

S $-9.10360600-3.30944200-0.19020200$

S $-8.09469600-0.59019500-2.50940600$

S -7.50238300 -3.88164900 4.21910900

$\mathrm{Au} 1.82942800-1.236675003 .10172600$

Au 2. 63613000 2. $15167600-2.34653000$

$\mathrm{Au} 4.02847800 \quad 0.24952600 \quad 2.19665700$

$\mathrm{Au} 4.43965200 \quad 0.61363000-0.74985100$

$\mathrm{Au} 4.27515500$ 3.44260000 -0.48333400

Au $4.00287400-2.526983001 .85256800$

$\mathrm{Au} 4.84583600$-2. $23615900-1.18051700$

Au $6.42119500-0.91000000 \quad 0.68135800$

$\mathrm{Au} 6.72447300-0.41511300-2.05992100$

S $6.241312004 .81184000-1.03824900$

Au 7. 19574200 2.91931200 -2. 11466100

S $8.149532001 .10717500-3.32476900$

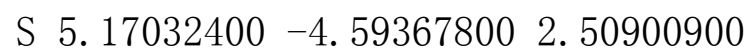

Au 7. $03641900-4.001445001 .18085800$

S 2. 49644600 2. 142533005.51351200

$\mathrm{Au} 3.67348300 \quad 0.08170600$ 5. 49032700 
S 4.78431900 -2.01637100 5.69550300

S $3.76383700-0.80053200-4.69502400$

$\mathrm{Au} 5.65724700$-2. 17092300 -4. 39576200

$\begin{array}{lll}\text { S } 8.06575600 & -0.77402300 & 2.49922400\end{array}$

S 9. $04356300-3.40758900 \quad 0.05550300$

$\mathrm{Au}$ 6. $37001500-1.39728900$ 4. 03968900

$\mathrm{Au} 8.16157900-3.37550600-2.14351800$

S $7.57727700-3.56715200-4.43203400$

S -6. 303079004.692329001 .18522700

$\mathrm{Au}-7.15974600 \quad 2.78414800 \quad 2.31691200$

S $-5.602449002 .23838000-2.64465500$

$\mathrm{Au}-4.11149800$ 4. $04588200-2.33536900$

$\mathrm{Au}$ 4. 14520900 3. 91792300 2. 51907900

$\begin{array}{llll}\text { S 5. } 52955900 & 2.08808600 & 2.71509400\end{array}$

H $6.87721000 \quad 4.67346800 \quad 0.16158900$

H 9. $25337900 \quad 0.95997000-2.53518600$

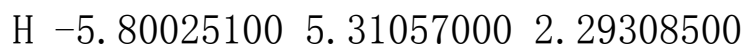

H -7. $32099900 \quad 0.92277300 \quad 4.50914200$

H 6.13607900 2. $16279500 \quad 1.44682200$

$\begin{array}{llll}\text { H } 3.80046800 & 6.75167000 & 2.23973900\end{array}$

H 2. $212557005.03000200-2.56981000$

$\mathrm{H}-2.19549900 \quad 4.86762600 \quad 2.83279100$

H -3. $802050006.85544100-1.88974000$

$\mathrm{H}-6.147346002 .27446200-1.39140000$

$\mathrm{H}-3.532652003 .19502100-5.03999700$

$\mathrm{H}-5.59380300-1.36123700-6.84502500$

$\mathrm{H}-7.97976700 \quad 0.76185500-2.67724600$

H 3. 11432600 2. $10170400-5.23787100$

$\mathrm{H}-1.31127700-1.00609500-6.05670300$

H $2.99398400-1.72519500-5.33785400$

H $6.89785300-4.74565700-4.30718300$

H $9.58129400-4.66067300-0.03305300$

H $4.52260000-5.402245001 .61658400$

H $4.77261000-5.16182200-1.05461300$

H $0.21877500-5.27700600-1.60080700$

H $-0.20250100-5.35173300 \quad 1.29939800$

$\mathrm{H}-4.75873100-5.21477100 \quad 0.71684200$

$\mathrm{H}-5.63025800-4.12819100-3.75987700$

$\mathrm{H}-9.66785500-4.55422800-0.18220800$

$\mathrm{H}-6.78173500-5.011410003 .95353500$

H $-3.00382000-1.91465400 \quad 5.27033000$

$\begin{array}{llll}\text { H } 7.95811500 & 0.56911600 & 2.73328500\end{array}$

H $5.54451100-1.692486006 .78351200$ 
$\begin{array}{lll}\text { H } 3.54180300 & 2.95178500 & 5.16057800\end{array}$

H $1.30539400-1.31242500 \quad 5.99006100$

H -3. $10602300 \quad 1.85032700$ 5. 32517600 